\title{
Currency Risk Hedging With Time-Varying Correlations
}

\author{
Binbin Guo, (E-mail: guo@ cats.ucsc.edu), University of California, Santa Cruz
}

\begin{abstract}
This paper studies currency risk hedge when volatilities and correlations of forward currency contracts and underlying assets returns are all time-varying. A multivariate GARCH model with time-varying correlations is adopted to fit the dynamic structure of the conditional volatilities and correlations. The conditional risk-minimizing hedge strategies are estimated for an international portfolio of the US, UK and Switzerland stocks, for the period of February of 1973 to March of 2002. The empirical results show that the optimal dynamic hedging strategies can capture partially the currency fluctuations, and greatly reduce the currency risk and enhance the risk-adjusted returns of the portfolio with significant foreign currency exposures.
\end{abstract}

\section{Introduction}

$c$

urrency risk has become an important component of a foreign investment, as globalization and integration of financial markets worldwide gained momentum over the past two decades. Currency risk is the volatility of the exchange rate of one currency for another i.e. that the currency in which an investment is made will decline in value, relative to the investor's currency. The differences between volatilities measured in foreign currency and those measured in U.S. dollars (for a investor in the U.S.) represent the contribution of currency risk to total portfolio risk. Since the end of the Bretton Woods system in February 1973 when the exchange rates of all the industrial countries were set free to float independently, exchanges rates of developed countries have been highly volatile and correlations between exchange rates have also been changed dramatically over time. While a part of currency risks (idiosyncratic risks) get diversified away in an international portfolio invested in many currencies, systematic currency risks remain. Both theoretical and empirical evidence suggest that the most effective way of reducing systematic currency risks is hedging through future or forward contracts (Solnik, 1974, Black, 1990). Eun and Resnick (1988) and Glen and Jorion (1993) also show that currency hedging improves the risk-return performance of international portfolio as well as reducing risks. However, most of the academic research has considerable disagreement on the strategies that global investors should use to hedge currency risk. Specifically, there is little consensus on how much to hedge or optimal hedge ratios.

Extensive research in the literature has been focused on developing optimal hedging strategies. In early theoretical approach, Solnik (1974) develops an international asset pricing model (IAPM) and conclude that all investors should hold the same portfolio of risky assets, in which currency risks are optimally hedged through the inclusion of forward contracts. Black (1990) extends Solnik's IAPM model and suggests that there exists a "universal" hedge ratio that is optimal to all investors. Anderson and Danthin (1981) derive a risk-minimizing hedge ratio using unconditional variance-covariance matrix between cash and forward price changes. Briys and Solnik (1992), and Lioui and Poncet (2002) derives the optimal utility-maximizing demand of forward exchange contracts to hedge currency risk under a continuous-time framework. Major drawback for all these models is that the hedge ratios are derived based on a strong assumption that the volatilities of, as well as correlations between, changes in underlying assets and currency forward prices remain constant over time.

$\overline{\text { Readers with comments }}$ or questions are encouraged to contact the author via email. 
Overwhelming empirical evidences show that the volatilities, as well as the correlations, of spot and forward exchange price change are time-varying. See Kroner and Sultan (1991), Sheedy (1998), among many others. Ignoring such time-varying variance-covariance structure in estimating currency hedging demand tends to over-estimate the number of futures contracts required to hedge the cash position and leads to a sub-optimal mix in the composition of the hedged portfolio (Gagnon et al, 1998). In order to capture the dynamic structures of conditional second moments of underlying asset and exchange forward price changes, recent empirical work has been centered on developing timevarying hedging ratios utilizing conditional heteroskedasticity modeling techniques. Typically, autoregressive conditional heteroskedasticity (ARCH) and its variants (Engle, 1982, Bolleslev, 1986) are typically employed to model the dynamic structures of the conditional second moments.

Along this direction, Kroner and Claessens (1991) illustrate using GARCH techniques can improve hedging currency risk associated with external debt. Kroner and Sultan (1993) apply the constant-correlation bivariate GARCH model to currency hedge. While conditional variances of underlying assets and exchange forward prices change are changing over time, the conditional correlations for currency markets are assumed to be constant in order for the variance-covariance matrix to be positive definite, as proposed in Bolleslev (1990). This constant-correlation approach has been widely applied because of its computational simplicity. But, financial data in exchange rates again show strong evidence that the assumption of constant correlation is violated for currency markets even after taking into account of volatility clustering (Sheedy, 1998, Tse and Tsui, 2002). To capture time-varying feature in conditional correlations between exchange rates, Tong (1996) and Gagnon et al (1998) adopt the BEKK multivariate GARCH (MGARCH) procedure of Engle and Kroner (1995). The BEKK algorithm permits time-variation in the conditional covariance while it ensures the condition of a positive-definite conditional variance-covariance matrix. However, as for currency markets, Sheedy found that "the BEKK specification is not effective in eliminating structure in correlation, despite the fact that it is designed to do so". Bera et al (1997) also report that the BEKK model does not perform well in the estimation of optimal hedge ratios. Convergence problem with BEKK procedure (Lien et al 2001) further limits the application of this approach. Most recently, Tse and Tsui (2002) extend the multivariate GARCH models to incorporate time-varying correlations as BEKK model does and yet retain the appealing feature of satisfying the positive-definite condition and keeping computational simplicity as found in constant-correlation model. In this paper, we adopt Tse and Tsui's MGARCH specification to model time-varying conditional variance-covariance matrix in the estimation of optimal hedge ratios.

The objectives of this paper are two-folded. First, this paper develops a risk-minimizing model to estimate hedging ratios when volatilities and correlation of forward currency contracts and underlying assets are all timevarying. A VAR-MGARCH model is specified to estimate the conditional variance-covariance matrix. Secondly, the model and estimation procedure are applied to an international portfolio of the US, UK and Switzerland stocks, for the period of February of 1973 to March of 2002. Our empirical examples show that the optimal dynamic hedging strategies can capture partially the currency fluctuations. The optimal time-varying strategies greatly reduce the total risk and enhance the risk-adjusted returns on a portfolio with significant foreign currency exposures.

The rest of the paper proceeds as follows. Section 2 derives a conditional risk-minimizing model to estimate optimal hedge ratios for an international portfolio hedged with forward contracts. Section 3 specifies econometric model to estimate conditional variance-covariance matrix. Data and summary statistics are described in Section 4. Section 5 documents the empirical estimation, Section 6 evaluates the hedging performance, and section 7 concludes the paper.

\section{Risk-Minimizing Hedge Model}

Consider an agent who has investment in domestic asset of unit $v_{0, t-1}$, and $\mathrm{N}$ foreign assets of unit $v_{k, t-1}$ ( $\mathrm{k}=1$ to $\mathrm{N}$ ) at beginning of investment period $\mathrm{t}-1$. The price for domestic asset is denoted as $P_{0, t-1}$ and foreign assets as $P_{k, t-1}(\mathrm{k}=1$ to $\mathrm{N})$ in foreign currency. Let spot rates for currency $k$ be $E_{k, t-1}$ expressed as the domestic currency per unit of the foreign currency, for $\mathrm{k}=1$ to $\mathrm{N}$. We can simplify the notation by defining: 


$$
S_{k, t-1}=P_{k, t-1} E_{k, t-1} \text {, for } \mathrm{k}=1 \text { to } \mathrm{N},
$$

which simply represent foreign asset prices in domestic currency. Without loss of generality, let domestic spot rate be 1. Then the initial investment of the agent is equal to $\sum_{k=0}^{N} v_{k, t-1} S_{k, t-1}$ and currency exposure for this un-hedged portfolio is $\sum_{k=1}^{N} v_{k, t-1} S_{k, t-1}$ at beginning of investment period t-1. These unified notations permit any foreign investment instruments to be defined. In our empirical example in Section 4, foreign assets are assumed to be cash stock indices. If foreign assets include cash currency holding itself, $P_{k, t-1}$ can be simply set to 1 to keep the notations unchanged.

In order to hedge the corresponding spot currency risks, the agent holds short positions of $-\phi_{k, t-1}$ ( $\mathrm{k}=1$ to $\mathrm{N})$ units in the underlying $k$ th foreign currency forward. The hedging ratio on currency $k, h_{k, t-1}$, is defined as follows:

$$
h_{k, t-1}=\frac{\phi_{k, t-1}}{v_{k, t-1} P_{k, t-1}},
$$

It is noted that $h_{k, t-1}=1$ is full hedging, and $h_{k, t-1}=0$ is no hedging. In a static model, this ratio stays constant over time. In our dynamic model, $h_{k, t-1}$ change over time due to the changes in variance-covariance between cash and forward rates over time.

The returns on underlying assets over the investment period is measured in a standard way:

$$
r_{k, t}^{s}=\frac{S_{k, t}-S_{k, t-1}}{S_{k, t-1}}
$$

then return on an unhedged portfolio is given by:

$$
r_{p, t}^{u}=\sum_{k=0}^{N} w_{k, t-1} r_{k, t}^{s}
$$

where $w_{k, t-1}=\frac{v_{k, t-1} S_{k, t-1}}{\sum_{k=0}^{N} v_{k, t-1} S_{k, t-1}}$ is the weight of initial investment in asset $k$.

A forward contract is an agreement to buy or sell foreign currency at a certain time in the future, but require no initial investment. Therefore, a standard way of defining rate of return is not applicable to forward contracts. However we can define a rate of return associated with forward contracts by measuring the difference between the return on portfolio with and without forward contracts. 
The return on a portfolio hedged with currency forward contracts can be derived as follows:

$$
\begin{aligned}
r_{p, t} & =\frac{\sum_{k=0}^{N} v_{k, t-1}\left(S_{k, t}-S_{k, t-1}\right)-\sum_{k=1}^{N} \phi_{k, t-1}\left(F_{k, t}-F_{k, t-1}\right)}{\sum_{k=0}^{N} v_{k, t-1} S_{k, t-1}} \\
& =\sum_{k=0}^{N} w_{k, t-1} r_{k, t}^{S}-\sum_{k=1}^{N} h_{k, t-1} w_{k, t-1} r_{k, t}^{f},
\end{aligned}
$$

where $r_{k, t}^{f}=\frac{F_{k, t}-F_{k, t-1}}{E_{k, t-1}}$ is a "normalized" return on a long forward contract. In this notation, initial cash currency exposure $E_{k, t-1}$ is used as the "base" instead of forward rate $F_{k, t-1}$. This "normalized" return can be further decomposed as the follows:

$$
\begin{aligned}
r_{k, t}^{f} & =\frac{F_{k, t}-F_{k, t-1}}{E_{k, t-1}}=\frac{F_{k, t}-E_{k, t}}{E_{k, t}} \frac{E_{k, t}}{E_{k, t-1}}-\frac{F_{k, t-1}-E_{k, t-1}}{E_{k, t-1}}+\frac{E_{k, t}-E_{k, t-1}}{E_{k, t-1}} \\
& =D_{t} \frac{E_{k, t}}{E_{k, t-1}}-D_{t-1}+\frac{E_{k, t}-E_{k, t-1}}{E_{k, t-1}}
\end{aligned}
$$

where $D_{t}$ and $D_{t-1}$ are forward discount or premium at time $\mathrm{t}$ and $\mathrm{t}-1$ respectively. So, $r_{k, t}^{f}$ is approximately equal to the sum of the change in forward discount/premium and return on spot exchange rate of underlying currency. If we further assume that interest rate parity hold, forward discount/premium should be equal to the interest rate differentials of foreign and domestic countries. It is clear that both change in interest rate differentials and underlying spot exchange rate fluctuation will affect the $r_{k, t}^{f}$, and in turn the optimal hedge ratios.

Let $X_{t-1}=\left(w_{0, t-1}, w_{1, t-1}, \ldots, w_{N, t-1}, h_{1, t-1} w_{1, t-1}, h_{2, t-2} w_{2, t-2}, \ldots, h_{N, t-1} w_{N, t-1}\right)^{\prime}$ be the weight vector, and $R_{t}=\left(r_{0, t}^{s}, r_{1, t}^{s}, \ldots, r_{N, t}^{s}, r_{1, t}^{f}, r_{2, t}^{f}, \ldots, r_{N, t}^{f}\right)^{\prime}$ be the return vectors.

The return on a hedged portfolio can be re-expressed in a standard matrix notation as follows:

$$
r_{p, t}=X_{t-1}^{\prime} R_{t}
$$

We first consider a utility-maximizing problem. Suppose that the agent is to select optimal weights on spot assets and hedging ratios on forward contracts to maximize his quadratic form utility: 


$$
\begin{aligned}
& \underset{h_{k, t-1}, w_{k, t-1}}{\operatorname{Max}}\left[E\left(r_{p, t} \mid I_{t-1}\right)-\frac{1}{2} \lambda \operatorname{Var}\left(r_{p, t} \mid I_{t-1}\right)\right]=\underset{h_{k, t-1}, w_{k, t-1}}{\operatorname{Max}}\left[E\left(r_{p, t} \mid I_{t-1}\right)-\frac{1}{2} \lambda X_{t-1}^{\prime}\left(\Omega_{t} \mid I_{t-1}\right) X_{t-1}\right] \\
& \text { Subject to: } \sum_{k=0}^{N} w_{k, t-1}=1 .
\end{aligned}
$$

where $I_{t-1}$ is the information available at time $t$. where the conditional variance-covariance matrix $\Omega_{t} \mid I_{t-1}=\Sigma_{t}$ can be partitioned as $\Sigma_{t}=\left[\begin{array}{cc}\Sigma_{s s, t} & \Sigma_{s f, t} \\ \Sigma_{s f, t}^{\prime} & \Sigma_{f f, t}\end{array}\right]_{(2 N+1) \times(2 N+1)}$,

where $\Sigma_{s s, t}$ is a $(N+1) \times(N+1)$ conditional variance-covariance matrix of cash rates of returns on both domestic and foreign assets, $\Sigma_{f f, t}$ is a $N \times N$ conditional variance -covariance matrix of the returns on currency forward contracts. $\Sigma_{s f, t}$ is a $(N+1) \times N$ conditional variance-covariance matrix between the returns on cash assets and currency forward contracts.

While this standard utility-maximization problem is easily derived, the conditional optimal solutions could be very instable due to the low predict power on conditional expected returns on currency forward contracts. In our empirical examples in section 4, all conditional mean parameters are insignificant. It suggests that forward rates may follow martingale processes, $E\left(r_{k, t}^{f} \mid I_{t-1}\right)=0$. Alternatively, we focus on a risk-minimizing model. Our agent is assumed to minimize the risk of his total positions which is subject to exchange rate fluctuations:

$$
\begin{aligned}
& \operatorname{Min}_{h, t-1}, w_{k, t-1}\left(\operatorname{Var}\left(r_{p, t} \mid I_{t-1}\right)={\underset{h}{k, t-1}, w_{k, t-1}}_{h_{t-1}}\left\{\Omega_{t}^{\prime} \mid I_{t-1}\right) X_{t-1}\right\} \\
& \text { Subject to: } \sum_{k=0}^{N} w_{k, t-1}=1 .
\end{aligned}
$$

It is noted that if the underlying asset prices and the forward rates are all following martingale processes optimal solutions to utility-maximization and risk-minimizing are equivalent, as indicated in Gagnon (1998).

In both Models (6) and (7), we assume that hedging decision is part of investment decision. We have total $\mathrm{N}+1$ weights for spot assets and $\mathrm{N}$ hedging ratios for forward contracts to be determined by system. It is clear that in our optimization process, forward contracts are treated equally as other asset class instruments. To investigate pure hedging effects, we assume investment decision has been made prior to hedging decision. Given the weights on underlying assets $w_{k, t-1}$, the agent chooses optimal hedging ratios to minimize the risk. We also consider short selling restrictions the agent faces when they make hedging decision. The optimal short positions on forward currency derived on above models based on estimated conditional variance-covariance matrix could be extremely large. The hedge ratios could be well over unity. Therefore, forward currency is restricted to selling short no more than the exposure of the underlying currency, i.e., $h_{k, t-1} \leq 1$, for $\mathrm{k}=1$ to $\mathrm{N}$. Therefore, we form our agent risk-minimizing problem as follows: 


$$
\begin{aligned}
& \underset{h_{k, t-1}}{\operatorname{Min}}\left(\operatorname{Var}\left(r_{p, t} \mid I_{t-1}\right)=\underset{h_{k, t-1}}{\operatorname{Min}}\left\{X_{t-1}^{\prime}\left(\Omega_{t} \mid I_{t-1}\right) X_{t-1}\right\}\right. \\
& \text { Subject to: } \sum_{k=0}^{N} w_{k, t-1}=1 \text { and } h_{k, t-1} \leq 1, \text { for } \mathrm{k}=1 \text { to } \mathrm{N}
\end{aligned}
$$

This optimization problem with inequality constraints can be solved by Kuhn-Tucker method. While numerical solution is easily implemented, analytical solution is referred to any standard operation research literature for brevity of the paper.

\section{Econometric Specification}

Having established our hedging optimization models in last section we are now in the position to specify our econometric model in order to estimate conditional second moments. Our econometric model for return series $R_{t}=\left(r_{0, t}^{s}, r_{1, t}^{s}, \ldots, r_{N, t}^{s}, r_{1, t}^{f}, r_{2, t}^{f}, \ldots, r_{N, t}^{f}\right)^{\prime}$ can be specified as a VAR-GARCH process:

$$
r_{t}=\mu+\sum_{i=1}^{s} \alpha_{i} r_{t-i}+u_{t}
$$

where $E\left(u_{t}\right)=0, \operatorname{Var}\left(u_{t}\right)=\sigma^{2} I$, and $\operatorname{Var}\left(u_{t} \mid I_{t-1}\right)=\Sigma_{t}$.

$\Sigma_{t}$ is specified as the process of MGARCH with time-varying correlations introduced by Tse and Tsui (2002).

Following Tse \&Tsui (2002), the conditional variance elements of $\Sigma_{t}$ is denoted as $\sigma_{i t}^{2}$ for $\mathrm{i}=1,2, . .2 \mathrm{~N}$, and the conditional covariance elements as $\sigma_{i j t}$, where $1 \leq i<j \leq 2 N$. Let $D_{t}$ be the $(2 N+1) \times(2 N+1)$ diagonal matrix where ith diagonal element is $\sigma_{i t}^{2}$. Then we have:

$$
\Sigma_{t}=D_{t} \Gamma_{t} D_{t}
$$

with $\Gamma_{t}=\left\{\rho_{i j t}\right\}_{(2 N+1) \times(2 N+1)}$ is the correlation matrix of $\varepsilon_{t}$.

Conditional variance term follows a univariate $\operatorname{GARCH}(\mathrm{p}, \mathrm{q})$ model given by the equation:

$$
\sigma_{i t}^{2}=a_{i}+\sum_{m=1}^{p} b_{i m} \sigma_{i, t-m}^{2}+\sum_{m=1}^{q} c_{i m} \varepsilon_{i, t-m}^{2}, \quad \mathrm{I}=1,2, \ldots 2 \mathrm{~N}+1,
$$

where $a_{i}, b_{i m}, c_{i m}$ are nonnegative, and $\sum_{m=1}^{p} b_{i m}+\sum_{m=1}^{q} c_{i m}<1$, for $\mathrm{I}=1,2, \ldots .2 \mathrm{~N}+1$.

Conditional correlation matrix $\Gamma_{t}$ is generated from the recursion:

$$
\Gamma_{t}=\left(1-\lambda_{1}-\lambda_{2}\right) \Gamma+\lambda_{1} \Gamma_{t-1}+\lambda_{2} \Psi_{t-1},
$$


where $\lambda_{1}+\lambda_{2} \leq 1, \Gamma=\left\{\rho_{i j}\right\}_{(2 N+1) \times(2 N+1)}$ is a constant positive definite parameter matrix with unit diagonal elements. $\Psi_{t}=\left\{\psi_{i j t}\right\}_{(2 N+1) \times(2 N+1)}$ with

$\psi_{i j, t-1}=\frac{\sum_{m=1}^{M} \varepsilon_{i, t-m} \varepsilon_{j, t-m}}{\left(\left(\sum_{m=1}^{M} \varepsilon_{i, t-m}^{2}\right)\left(\sum_{m=1}^{M} \varepsilon_{j, t-m}^{2}\right)\right)^{1 / 2}}, \quad 1 \leq i<j \leq 2 N+1, M \geq 2 N+1$.

where $\varepsilon_{i t}$ is the ith element of the standardized residual vector $\varepsilon_{t}=D_{t}^{-1} u_{t}$. As $\Psi_{t}$ is a positive definite with unit diagonal elements, $\Gamma_{t}$ will also become a well-defined correlation matrix. With assumption of conditional normality, $u_{t} \mid I_{t-1} \sim N\left(0, D_{t} \Gamma_{t} D_{t}\right)$, the conditional maximum likelihood estimation procedure can be employed to estimate all parameters. This time-varying correlation MGARCH model specification (Tse and Tsui, 2002) incorporates timevarying correlations and yet retains the appealing feature of satisfying the positive-definite condition and keeping computational simplicity as found in constant-correlation MGARCH model.

\section{Data Description}

In our empirical analysis we considered three markets, the United States as domestic market, and the United Kingdom and Switzerland as foreign markets. All data are monthly observations from February of 1973 through May of 2002, covering the entire period of the post Bretton Woods system in that the exchange rates of all the industrial countries are free to float independently. Unlike many previous work in literature in that they use daily data, we use monthly data to conduct hedging strategies. This seems closer to hedging practice where rebalancing hedging positions rarely take place in daily bases. Monthly spot and one-month forward exchange rates, Great Britain Sterling (GBP) forward and Swiss Franc (SWF) forward, were obtained from the OECD-MEI database. All exchange rates are expressed as the U.S. dollar (domestic currency) per unit of the foreign currencies.

To examine pure hedging effect, the underlying asset weights for unhedged portfolio has been ad hoc determined prior to hedging decision. In our empirical examples, the portfolios considered compose of the United States stocks as domestic assets, and United Kingdom and Switzerland stocks as foreign assets. We construct three equity portfolios, (1) the US and the UK stock (denoted as US-UK portfolio), (2), the US and Switzerland stocks (USSW), and (3) the US, the UK and Switzerland stocks (US-UK-SW). Constant-mix strategies are adopted to form the unhedged portfolios. Without loss of generality, we assume that the compositions are 50\% each in US and UK stocks in US-UK portfolio, 50\% each in US and Switzerland stocks in US-SW portfolio, and 50\% in US stock and 25\% each in the UK and Switzerland stocks in US-UK-SW portfolio. Correspondingly, three hedging strategies are estimated: (1) hedging by short selling GBP forward in US-UK portfolio, (2) hedging by short selling SWF forward in US-SW portfolio, (3) Hedging by short selling both GBP and SWF forward in US-UK-SW portfolio.

Morgan Stanley Capital International (MSCI) country indices are used as the proxies to represent the stock returns of each country's equity market. MSCI country indices are all measured in local currency. We transfer the return data in local currency into returns in US dollar by following transformation:

$$
r_{k, t}^{S}(U S D)=r_{k, t}^{s}(\text { Local currency }) *\left(E_{k, t} / E_{k, t-1}\right)+\left(E_{k, t}-E_{k, t-1}\right) / E_{k, t-1} \text {, for k=GBP, SWK }
$$

or alternatively, 
$r_{k, t}^{s}(U S D)=r_{k, t}^{s}($ Local currency $)+\ln \left(E_{k, t} / E_{k, t-1}\right)$, where $r_{k, t}^{s}($ Local currency $)=\ln \left(P_{k, t} / P_{k, t-1}\right)$ in continuous compounding framework.

Table 1 reports summary statistics. The first two rows of the table show the unconditional sample means and standard deviations of all data series. All series show strong excess kurtosis, while skewness seems not strong except MSCI-UK index. Unconditional correlations are reported in Table 2. The low correlations between MSCI-US cash index and GBP forward/SWF forward indicates GBP forward/SWF forward can have strong diversification effects on portfolio as well as the hedging effects on portfolio's currency exposures. The high correlation between GBP forward and SWF forward implies a possible benefits using multivariate GARCH model instead of univariate and bivariate GARCH models.

\section{Table 1}

\section{Summary Statistics}

The returns on MSCI-US, MSCI-UK, and MSCI_SW are all in US dollar, which are derived from returns in local currency and spot exchange rates. E.g. return $r_{k, t}^{s}(U S D)=r_{k, t}^{s}(G B P) *\left(E_{G B P, t} / E_{G B P, t-1}\right)+\left(E_{G B P, t}-E_{G B P, t-1}\right) / E_{G B P, t-1}$. The returns on USD/GBP forward and USD/SWF forward are calculated as the "normalized" return described in section 2. E.g., $r_{G B P, t}^{f}=\frac{F_{G B P, t}-F_{G B P, t-1}}{E_{G B P, t-1}}$.

\begin{tabular}{lccccc}
\hline & MSCI-US & MSCI-UK(\$) & MSCI-SW(\$) & GBP-forward & SWF-forward \\
\hline Mean & 0.01040 & 0.01175 & 0.01115 & -0.00113 & 0.00240 \\
Standard Deviation & 0.04538 & 0.06952 & 0.05542 & 0.03083 & 0.03635 \\
Kurtosis & 2.02796 & 11.75931 & 1.53655 & 1.73901 & 0.60925 \\
Skewness & -0.28963 & 1.45450 & -0.03184 & 0.01754 & -0.02469 \\
\hline
\end{tabular}

Table 2

Unconditional Correlations

The returns on MSCI-US, MSCI-UK, and MSCI_SW are all in US dollar, which are derived from returns in local currency and spot exchange rates. E.g. return $r_{k, t}^{s}(U S D)=r_{k, t}^{s}(G B P) *\left(E_{G B P, t} / E_{G B P, t-1}\right)+\left(E_{G B P, t}-E_{G B P, t-1}\right) / E_{G B P, t-1}$. The returns on USD/GBP forward and USD/SWF forward are calculated as the "normalized" return described in section 2. E.g., $r_{G B P, t}^{f}=\frac{F_{G B P, t}-F_{G B P, t-1}}{E_{G B P, t-1}}$.

\begin{tabular}{|c|c|c|c|c|c|}
\hline & MSCI-US & MSCI-UK(\$) & MSCI-SW(\$) & GBP-forward & SWF-forward \\
\hline MSCI-US & 1.00000 & 0.52936 & 0.50701 & 0.00904 & $(0.05537)$ \\
\hline MSCI-UK(\$) & 0.52936 & 1.00000 & 0.57230 & 0.41319 & 0.19892 \\
\hline MSCI-SW(\$) & 0.50701 & 0.57230 & 1.00000 & 0.34067 & 0.47207 \\
\hline GBP-forward & 0.00904 & 0.41319 & 0.34067 & 1.00000 & 0.61305 \\
\hline SWF-forward & $(0.05537)$ & 0.19892 & 0.47207 & 0.61305 & 1.00000 \\
\hline
\end{tabular}

\section{Model Estimation}


Estimation results of conditional mean and variance-covariance parameters in the VAR-MGARCH model specified in Section 3 are reported in Table 3. We report here only statistically significant estimates. First, we look at conditional mean parameters. It shows that none of AR coefficients in SWF forward is significant, and only intercept of GBP forward is significant. It suggests that both GBP forward and SWF forward may evolve as either a martingale process or martingale with drift. While the MSCI-US return has no statistically significant time-varying terms, the MSCI-UK and MSCI-SW appear to be AR(2) processes. For the conditional second moments, all GARCH(1,1) coefficients are significant at $95 \%$ confidence level, as reported in Table 3.

Table 3

Estimation Results Of Conditional Mean And Variance-Covariance Parameters In VAR-MGARCH Models Conditional Gaussian maximum likelihood estimation procedure is used to estimate VAR-GARCH model: $r_{t}=\mu+\sum_{i=1}^{S} \alpha_{i} r_{t-i}+u_{t}$, where $E\left(u_{t}\right)=0, \operatorname{Var}\left(u_{t}\right)=\sigma^{2} I$, and $\operatorname{Var}\left(u_{t} \mid I_{t-1}\right)=\Sigma_{t}$, with $\Sigma_{t}=D_{t} \Gamma_{t} D_{t}$. Conditional variance term follows an univariate GARCH(p,q) model given by the equation $\sigma_{i t}^{2}=a_{i}+\sum_{m=1}^{p} b_{i m} \sigma_{i, t-m}^{2}+\sum_{m=1}^{q} c_{i m} \varepsilon_{i, t-m}^{2}, \quad \mathrm{i}=1,2, \ldots 2 \mathrm{~N}+1$, and conditional correlation matrix $\Gamma_{t}$ is generated from the recursion $\Gamma_{t}=\left(1-\lambda_{1}-\lambda_{2}\right) \Gamma+\lambda_{1} \Gamma_{t-1}+\lambda_{2} \Psi_{t-1}$ (Tse and Tsui, 2002). The standard errors are included in parentheses.

\begin{tabular}{|c|c|c|c|c|c|}
\hline & MSCI-US & MSCI-UK & MSCI-SW & GBP Forward & SWF Forward \\
\hline & 0.01046 & 0.01173 & 0.00807 & -0.00156 & \\
\hline \multicolumn{6}{|l|}{$\mu$} \\
\hline & $(0.00228)$ & $(0.00231)$ & $(0.00224)$ & $(0.00071)$ & \\
\hline \multirow[t]{2}{*}{$\alpha_{1}$} & & -0.04196 & & & \\
\hline & & $(0.0233)$ & & & \\
\hline \multirow[t]{2}{*}{$\alpha_{2}$} & & 0.11742 & 0.08221 & & \\
\hline & & $(0.0479)$ & $(0.0415)$ & & \\
\hline \multirow[t]{2}{*}{$\mathrm{a}$} & 0.00011 & 0.00013 & 0.00018 & 0.00002 & 0.00014 \\
\hline & $(0.00006)$ & $(0.00004)$ & $(0.00009)$ & $(0.00001)$ & $(0.00007)$ \\
\hline \multirow[t]{2}{*}{$\mathrm{b}$} & 0.06022 & 0.09989 & 0.12851 & 0.06640 & 0.09428 \\
\hline & $(0.02760)$ & $(0.02281)$ & $(0.04961)$ & $(0.02493)$ & $(0.04412)$ \\
\hline \multirow[t]{3}{*}{$\mathrm{c}$} & 0.88771 & 0.89783 & 0.80245 & 0.90992 & 0.80010 \\
\hline & $(0.06112)$ & $(0.02301)$ & $(0.06853)$ & $(0.03750)$ & $(0.10673)$ \\
\hline & US-UK-GBP & US-SW-SWF & US-UK-SW-GBP-SWF & & \\
\hline \multirow[t]{2}{*}{$\lambda_{1}$} & 0.93762 & 0.94710 & 0.97366 & & \\
\hline & $(0.00781)$ & $(0.00903)$ & $(0.01597)$ & & \\
\hline \multirow[t]{2}{*}{$\lambda_{2}$} & 0.03550 & 0.02556 & 0.01356 & & \\
\hline & $(0.00585)$ & $(0.00731)$ & $(0.00313)$ & & \\
\hline
\end{tabular}

The conditional volatility of USD/GBP forward, and conditional correlation between USD/GBP forward and USD/SWF forward estimated from MGARCH model from Table 3 are reported in Figures 1 and 2. The results show clearly that both volatility and correlation are changing over time. Similar time-varying properties for conditional correlations between USD/GBP forward and MSCI-UK cash index, and between USD/GBP forward and MSCI-SW 
cash index are also obtained, as reported in Figures 4. The conditional correlation between USD/GBP forward and MSCI-US cash index also changes over time as shown in Figure 3, even though they seems uncorrelated unconditionally as reported in Table 2 .

\section{Figure 1}

\section{Conditional Volatility Of USD/GBP Forward}

Conditional volatilities of the returns on USD/GBP forward are estimated using MGARCH model described in Section 3. All parameters estimated are displayed in Table 2. The returns on USD/GBP forward are calculated as the "normalized" return described in section 2. E.g., $r_{G B P, t}^{f}=\frac{F_{G B P, t}-F_{G B P, t-1}}{E_{G B P, t-1}}$.

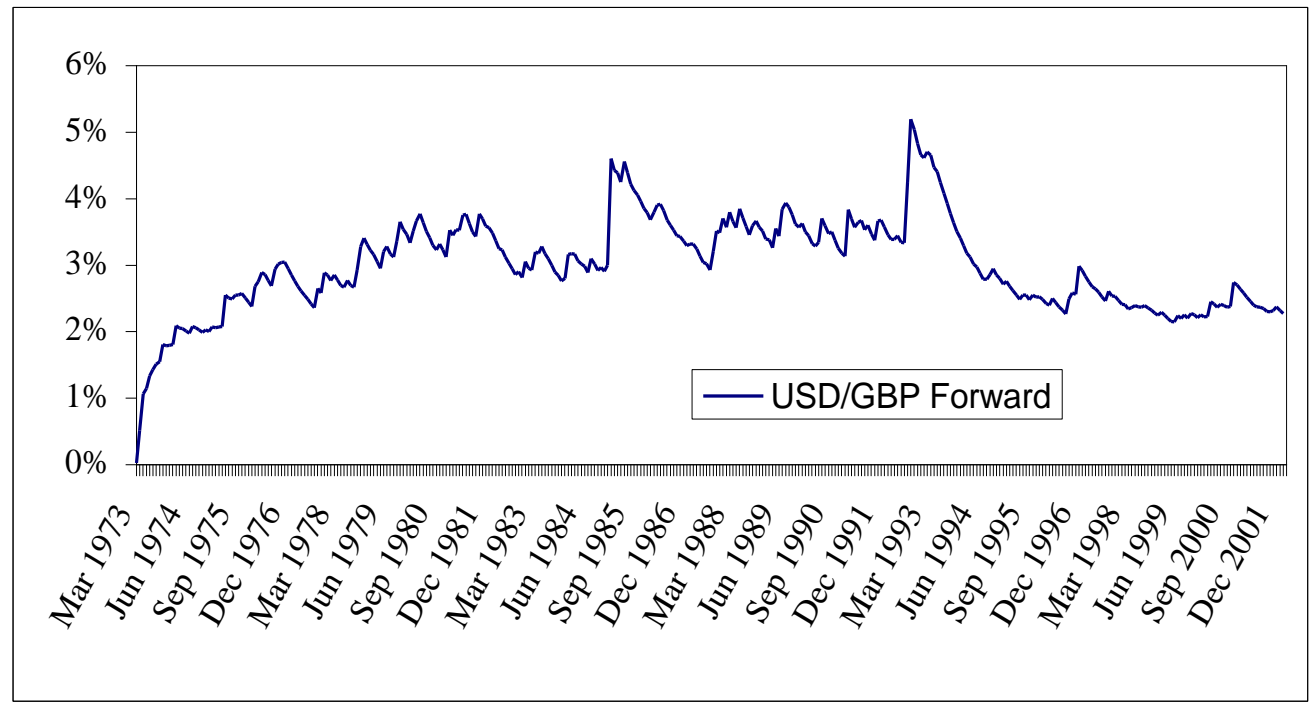

Figure 2

Conditional Correlation Between USD/GBP Forward And USD/SWF Forward

Conditional correlations between the returns on USD/GBP forward and USD/SWF forward are estimated using MGARCH model described in Section 3. All parameters estimated are displayed in Table 2. The returns on USD/GBP forward and USD/SWF forward are calculated as the

"normalized" return described in section 2. E.g., $r_{G B P, t}^{f}=\frac{F_{G B P, t}-F_{G B P, t-1}}{E_{G B P, t-1}}$. 


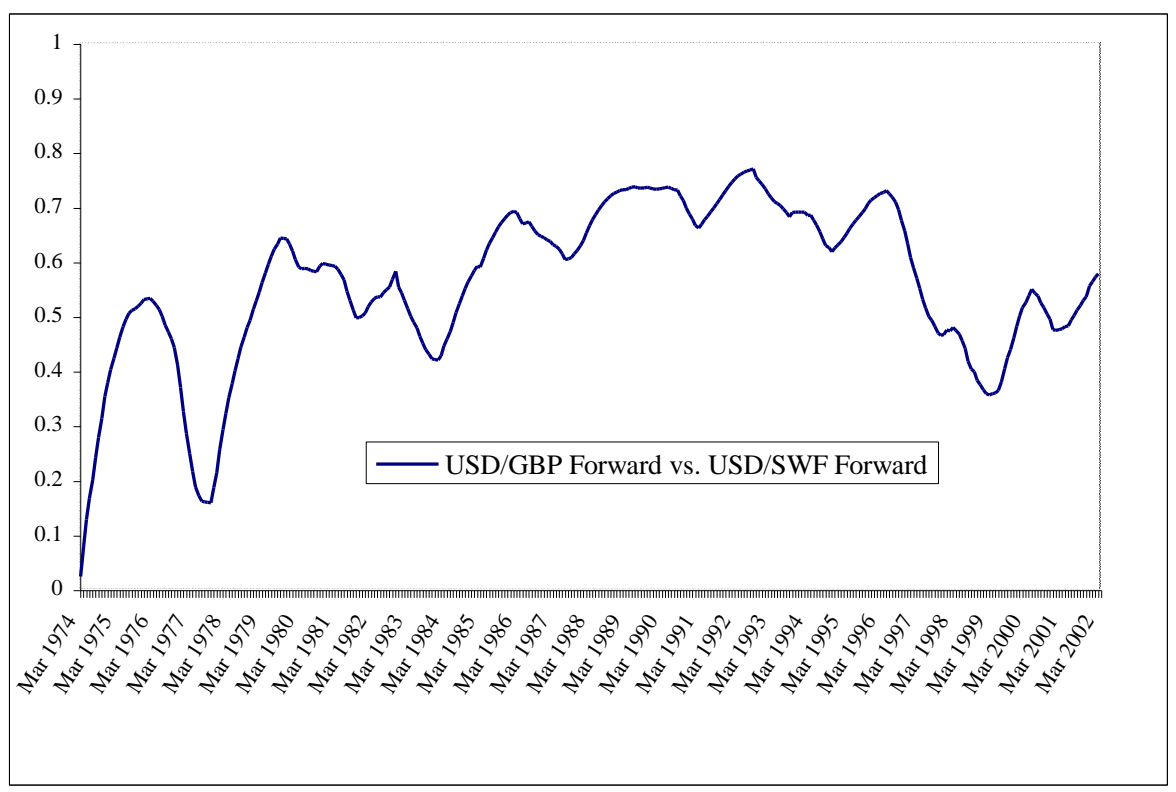

Figure 3 Conditional Correlation Between USD/GBP Forward And MSCI-UK Cash Index (In UD Dollar) 
Conditional correlations between the returns on USD/GBP forward and MSCI-US cash index are estimated using MGARCH model described in Section 3. All parameters estimated are displayed in Table 2. The returns on MSCI-US cash index are calculated as $r_{U S, t}^{s}=\frac{P_{U S, t}-P_{U S, t-1}}{P_{U S, t-1}}$, and the returns on USD/GBP forward are calculated as the "normalized" return, i.e., $r_{G B P, t}^{f}=\frac{F_{G B P, t}-F_{G B P, t-1}}{E_{G B P, t-1}}$.

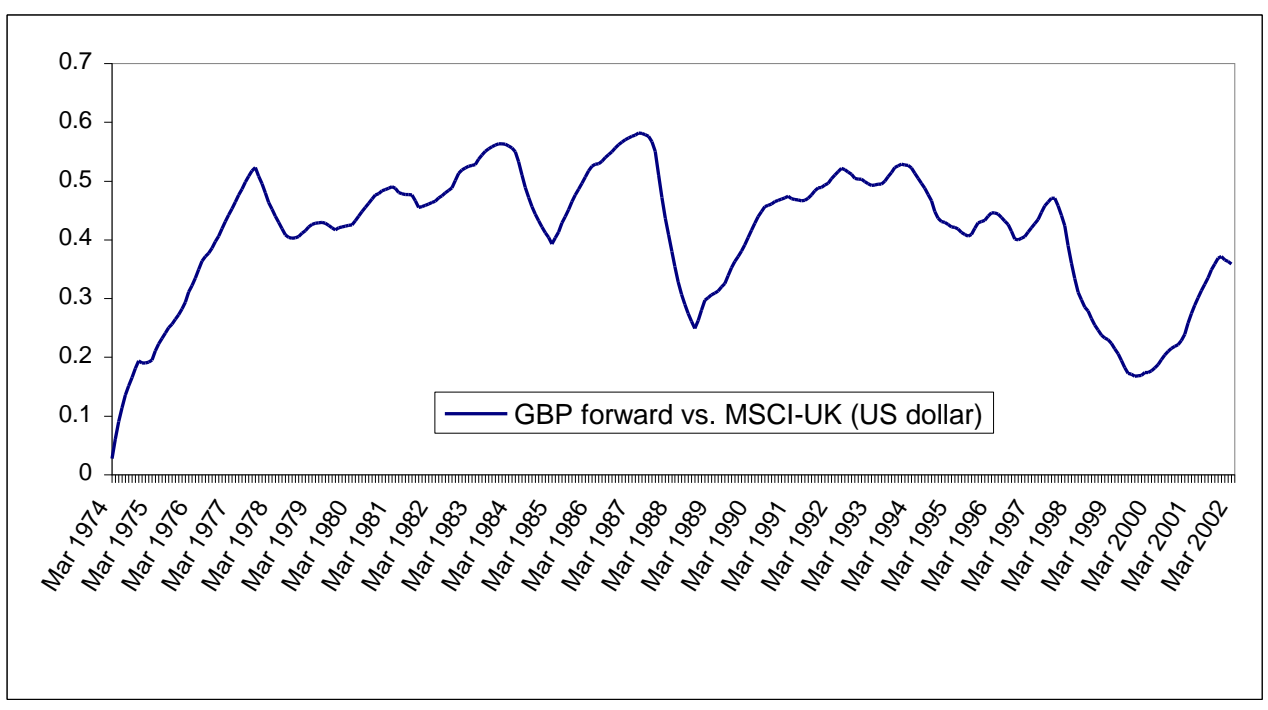

Figure 4

Conditional Correlation Between USD/GBP Forward And MSCI-US Cash Index

Conditional correlations between the returns on USD/GBP forward and MSCI-US cash index are estimated using MGARCH model described in Section 3. All parameters estimated are displayed in Table 2. The returns on MSCI-US cash index are calculated as $r_{U S, t}^{s}=\frac{P_{U S, t}-P_{U S, t-1}}{P_{U S, t-1}}$, and the returns on USD/GBP forward are calculated as the "normalized" return, i.e., $r_{G B P, t}^{f}=\frac{F_{G B P, t}-F_{G B P, t-1}}{E_{G B P, t-1}}$.

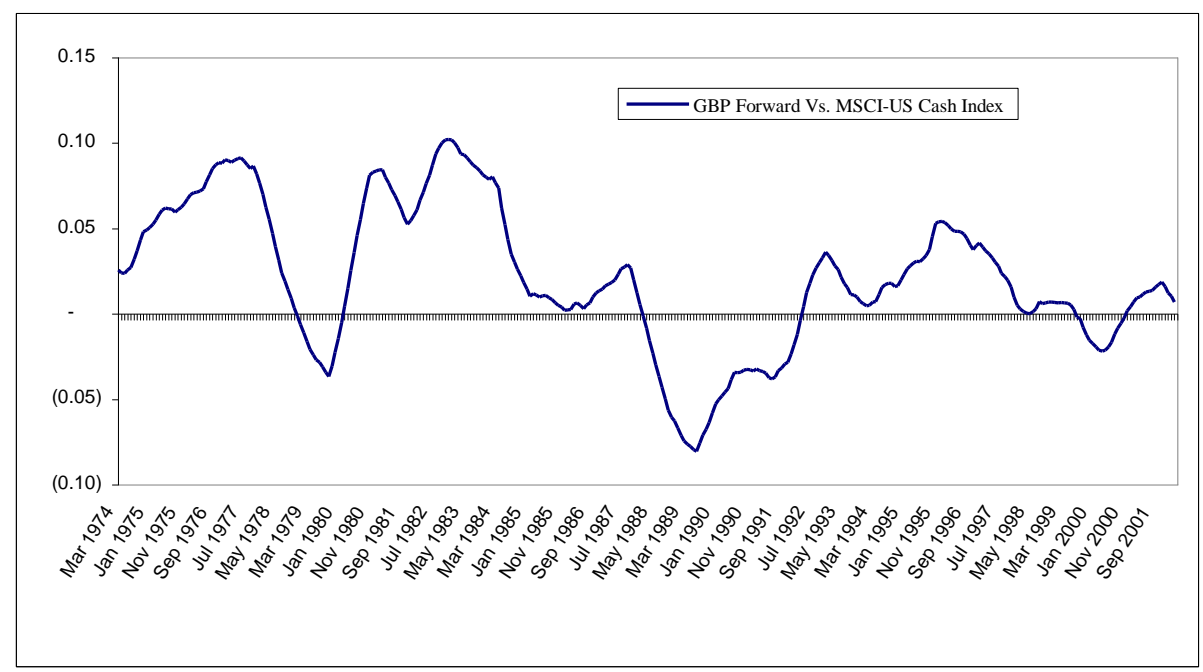




\section{Hedge Ratios And Hedging Performance}

The optimal time-varying hedging ratios in three equity portfolios are estimated based on risk-minimizing model (8) in Section 2. We assume that the compositions are 50\% each in US and UK stocks in US-UK portfolio, $50 \%$ each in US and Switzerland stocks in US-SW portfolio, and 50\% in US stock and 25\% each in the UK and Switzerland stocks in US-UK-SW portfolio. The results are reported in Table 3. In US-UK portfolio, average optimal hedge ratio for GBP forward contracts is 0.75 and standard deviation of 0.22 . Hedging is recommended all the time over the period 1973-2002.

In Figure 5, dynamic optimal hedging ratios for GBP forward in US-UK portfolio is illustrated. It appears that neither always-full-hedge nor never-hedge is optimal, and a portfolio with significant Sterling exposure should be hedged all the time. If ignoring speculative investment in GBP forward, hedge ratios are non-negative. Therefore, an effective dynamic hedging strategy is to fully hedgy when foreign currency is depreciating, and no hedgy or reducing hedging position when currency is appreciating. The effectiveness of our optimal hedge strategy is illustrated in Figure 6. Our optimal hedging strategy has successfully captured partial GBP rate fluctuations. In middle of 70s, GBP experiences years long depreciation, the hedgy ratios are increasing gradually to almost full hedging level. Hedging stays high during the period 1980-85 when GBP further depreciates. From 1987-90, GBP appreciates a bit, the hedgy ratio is moving down and stays low for this period. Starting year 2000, GBP depreciates continuously, the hedgy ratio again increases to reduce the risk from the GBP depreciation. The results are not particularly surprising. A GARCHtype model has reasonable power to predict future conditional variance and covariance that show strong clustering effects, as documented widely in the literature. We also observe that the conditional volatilities of exchange rate significant increase as that currency experiences sharp depreciations. This dynamic structure is estimated using multivariate GARCH model and incorporated through the risk-minimizing model into the hedging strategy.

Slightly different features are observed in SWF forward hedging in US-SW equity portfolio. The average hedge ratio for SWF forward decreases to 0.52, with standard deviation of 0.35, as shown in Table 3 . While a portfolio with exposure to Swiss Franc needs to be hedged $85 \%$ of the time, the dynamic hedge strategy suggests that $15 \%$ of the time should be unhedged or even long SWF forward contract (we denoted as "reverse hedge") to capture the gain from SWF appreciations during the sample period. This seems to be consistent with the fact that nominal Swiss Franc appreciates 86\%, while GBP depreciates 43\% during the period of 1973-2002. In our US-UK-SW equity portfolio, hedging positions on both GBP forward and SWF forward are reduced comparing with that in US-UK and US-SW portfolios. This occurs as a result of diversification effects. The total currency risk is lower by investing both UK and SW stocks than that investing in either UK or SW stocks only, as shown in Table 4. Therefore, the forward contracts are less demanded for hedging the currency risk.

Table 3

Hedge Ratios

The optimal hedgy ratios for GBP forward and SWF forward in three equity portfolios are estimated based on risk-minimizing model (8) in Section 2. The three portfolio compositions are 50\%-50\% in US-UK portfolio, 50\%-50\% in US-SW portfolio, and 50\%-25\%-25\% in US-UK-SW portfolio. Average ratios are arithmetic average of hedge ratios. Hedged percentage is the ratio of number of the month in that the hedge ratio is positive to total sample months. Unhedged percentage is the ratio of total number of unhedged month to total sample months. Reverse hedged percentage is the ratio of total number of negative-hedge-ratio month to total sample months.

\begin{tabular}{lcccc}
\hline & $\begin{array}{c}\text { GBP forward } \\
\text { In US-UK portfolio }\end{array}$ & $\begin{array}{c}\text { SWF forward } \\
\text { In US-SW portfolio }\end{array}$ & $\begin{array}{c}\text { GBP forward } \\
\text { In US-UK-SW portfolio }\end{array}$ \\
\hline Average & 0.75 & 0.52 & 0.63 & 0.29 \\
Standard deviation & 0.22 & 0.35 & 0.31 & 0.25 \\
Hedged & $100 \%$ & $85 \%$ & $83 \%$ & $69 \%$ \\
Unhedged & $0 \%$ & $9 \%$ & $12 \%$ & $16 \%$ \\
Reverse Hedged & $0 \%$ & $6 \%$ & $5 \%$ & $15 \%$ \\
\hline
\end{tabular}

Figure 5 


\section{Time-Varying Hedging Ratios}

The optimal hedgy ratios for GBP forward in US-UK equity portfolios are estimated based on risk-minimizing model (8) in Section 2. The portfolio compositions are $50 \%$ each in MSCI-US and MSCI-UK indices.

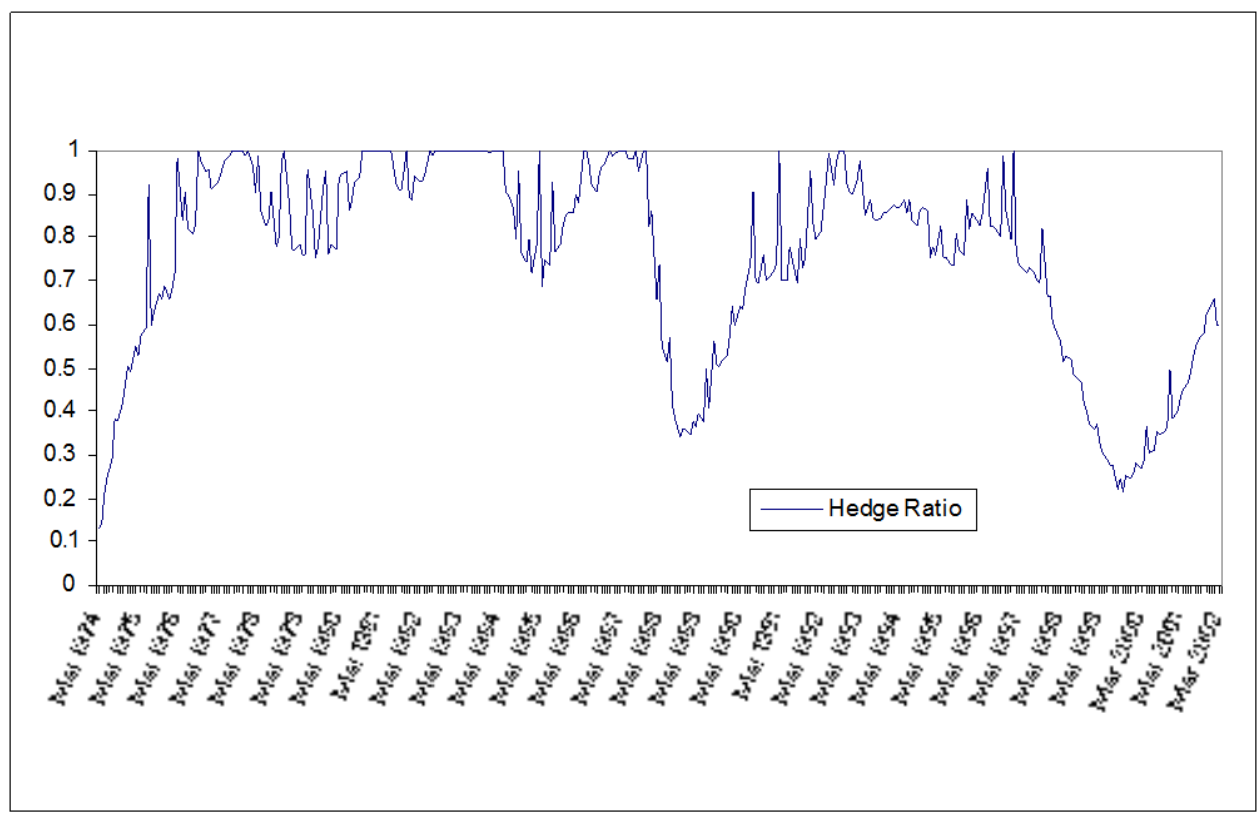

Figure 6

Hedging Ratios Vs. GBP Rates

The optimal hedgy ratios for GBP forward in US-UK equity portfolios are estimated based on risk-minimizing model (8) in Section 2. The portfolio compositions are 50\% each in MSCI-US and MSCI-UK indices. GBP rates denote the spot GBP/USD exchange rates.

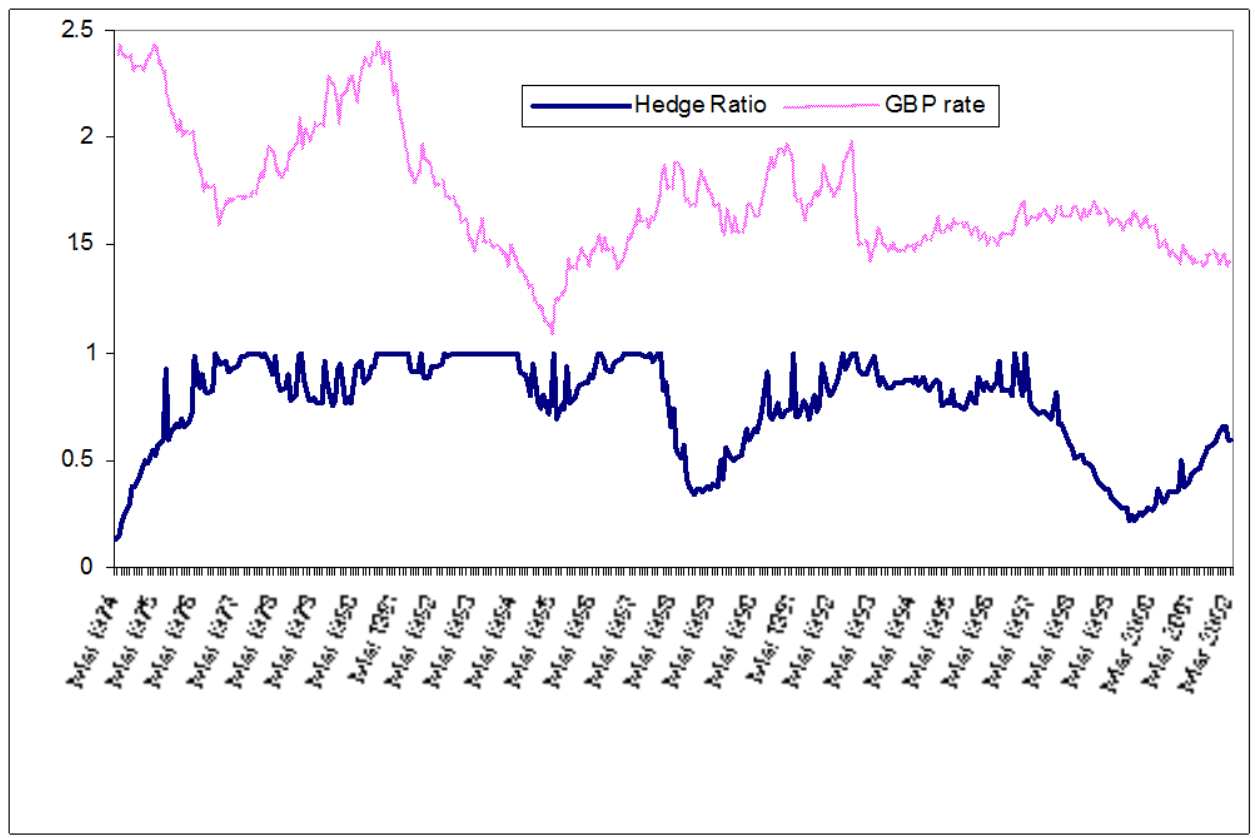


We now proceed to investigate the performances of our dynamic hedging strategies. In Table 4, we report the performances of our dynamic hedging strategy as well as three alternative static counterparts. Unhedged strategy is referred to $h_{k, t-1}=0$ strategy. Under this strategy, one never short or long forward contracts to cover currency risk. Fully hedged strategy is referred to $h_{k, t-1}=1$ strategy, and Universal hedge is referred to Black's (1990) "universal" hedging strategy. We adopt Black's empirical estimation, $h_{k, t-1}=0.7$ in this analysis. Currency risk is the incremental risk due to the currency exposure by investing foreign assets, MSCI-UK and MSCI-SW stock indices in our case. It is measured by the difference of portfolio risks with and without exchange rate fluctuations. By construction, currency risk is only around 5\% of the total portfolio risk in our examples. In order to examine the effectiveness of risk hedging strategies, we define the Risk Reduction as the percentage of incremental risk reduction due to the forward contracts to the total currency risk. We first look at the risk reduction effects. It shows that overall all strategies reduce significantly the currency risks. In both US-SW and US-UK-SW portfolios, universal-hedge strategy performs better than full-hedge strategy, while full-hedge does slightly better than universal-hedge in US-UK portfolio. On the other hand, our dynamic hedge appears to dominate all its static counterparts. In all three portfolio examples, the dynamic strategy can eliminate more than $90 \%$ of currency risk. Approximately, all currency risk in US-SW portfolio can be hedged away using SWF forward contracts.

Table 4

\section{Performance Of Hedged Portfolios}

Four strategies are: 1) Unhedged strategy, $h_{k, t-1}=0$,2) Universal hedge, $h_{k, t-1}=0.7$, (3) Fully hedged strategy, $h_{k, t-1}=1$ strategy, and (4) Dynamic hedge is referred to the time-varying optimal hedge estimated based on risk-minimizing model (8) in Section 2. The three portfolio compositions are 50\%-50\% in US-UK portfolio, 50\%-50\% in US-SW portfolio, and 50\%-25\%-25\% in US-UK-SW portfolio. Currency risk is the incremental risk due to the currency exposure by investing foreign assets, MSCI-UK and MSCI-SW stock indices in our case. It is measured by the difference of portfolio risks with and without exchange rate fluctuations. The Risk Reduction as the percentage of incremental risk reduction due to the forward contracts to the total currency risk. The US risk-free rate, 6.56\% during the period of 1973-2002, is used to calculated Shape ratios.

\begin{tabular}{|c|c|c|c|c|c|}
\hline & Mean return & Standard deviation & Currency risk & Risk reduction & Sharpe ratio \\
\hline & \multicolumn{5}{|c|}{ US-UK equity portfolio } \\
\hline Unhedged & $1.194 \%$ & $5.067 \%$ & $0.252 \%$ & $0.0 \%$ & 0.128 \\
\hline Universal hedged & $1.229 \%$ & $4.874 \%$ & $0.060 \%$ & $76.4 \%$ & 0.140 \\
\hline Fully hedged & $1.243 \%$ & $4.865 \%$ & $0.050 \%$ & $80.1 \%$ & 0.143 \\
\hline \multirow[t]{2}{*}{ Dynamic hedged } & $1.255 \%$ & $4.832 \%$ & $0.017 \%$ & $93.2 \%$ & 0.147 \\
\hline & \multicolumn{5}{|c|}{ US-SW equity portfolio } \\
\hline Unhedged & $1.152 \%$ & $4.365 \%$ & $0.141 \%$ & $0 \%$ & 0.139 \\
\hline Universal hedged & $1.075 \%$ & $4.239 \%$ & $0.015 \%$ & $89.2 \%$ & 0.125 \\
\hline Fully hedged & $1.057 \%$ & $4.272 \%$ & $0.048 \%$ & $65.8 \%$ & 0.120 \\
\hline \multirow[t]{2}{*}{ Dynamic hedged } & $1.133 \%$ & $4.226 \%$ & $0.002 \%$ & $98.4 \%$ & 0.139 \\
\hline & \multicolumn{5}{|c|}{ US-UK-SW equity portfolio } \\
\hline Unhedged & $1.173 \%$ & $4.489 \%$ & $0.158 \%$ & $0 \%$ & 0.139 \\
\hline Universal hedged & $1.147 \%$ & $4.354 \%$ & $0.024 \%$ & $85 \%$ & 0.138 \\
\hline Fully hedged & $1.135 \%$ & $4.375 \%$ & $0.045 \%$ & $72 \%$ & 0.135 \\
\hline Dynamic hedged & $1.159 \%$ & $4.338 \%$ & $0.007 \%$ & $95 \%$ & 0.141 \\
\hline
\end{tabular}


While currency risks are substantially reduced using hedge strategies, one concern is that returns on hedged portfolios may also decrease. In both portfolios with Swiss Franc currency exposure, all hedged portfolios have lower returns than the unhedged portfolios. This is partially due to the fact that nominal Swiss Franc appreciates $86 \%$ over the sample period, 1973-2002. The return on the portfolio hedged with short forward contracts is certainly lowered by short selling SWF forward contracts on average. However, it seems to be fair to examine the efficiency of risk reduction hedging based on the Sharpe ratios that measure the risk-return tradeoffs of the portfolios. We define an efficient hedging strategy based on the comparison of the Sharpe ratios of a portfolio with and without adding hedging positions on. Hedging is considered to be efficient if it increases or at least does not decrease the Sharpe ratio of a portfolio. Overall, there is no consistent evidence that universal-hedge and full-hedge are efficient. Both universal-hedge and full-hedge are efficient in US-UK portfolio, but not efficient in both portfolios with investing in SW stocks when the Sharpe ratios are substantially lower than the unhedged portfolios. On the other hand, the evidence on the efficiency of the dynamic strategy is more consistent. It is efficient in all three portfolios. In US-UK portfolio, it enhances the risk adjusted returns, from 0.128 to 0.147 , which is a substantial increase considering that the total currency risk contributes only less than 5\% of return variations, by construction, of the unhedged US-UK portfolio. The dynamic hedging strategy overperforms its static counterparts in terms of efficiency. One concern usually associated with time-varying investment strategy is the potential high transaction cost. However, in the currency hedging, transaction cost for currency forward contracts is very minimal, about $0.005 \%$ of face value of forward contracts, and above all, only monthly rebalancing is needed in our examples based on monthly data series.

Overall, our empirical examples show that the dynamic hedging strategy overperforms its static counterparts, adding an optimal combination of forward contracts based on a risk-minimizing model reduces more than $90 \%$ of currency risk and enhances the risk-return tradeoffs in all three equity portfolios.

\section{Conclusion}

This paper examines time-varying currency hedging strategies using currency forward contracts. While currency risk hedging is extensively studied in the literature, this paper establishes a simple conditional riskminimizing procedure based on MGARCH model with time-varying correlations. The MGARCH model specification (Tse and Tsui, 2002) adopted in this paper incorporates time-varying correlations and yet retains the appealing feature of satisfying the positive-definite condition and keeping computational simplicity as found in constant-correlation MGARCH model. Our empirical examples show that the optimal dynamic hedging strategies can capture partially the currency fluctuations, and overperforms its static counterparts. The optimal dynamic strategies greatly reduce the currency risk and enhance the risk-adjusted returns on the portfolio with significant foreign currency exposures.

\section{References}

1. Anderson, R. W., and J. Danthin, 1981, Cross hedging, Journal of Political Economy, 81, 1182-1196.

2. Bera, A. K., Garcia, P., and Roh, J. S., 1997, Estimation of Time-Varying Hedging Ratios for Corn and Soybeans: BGARCH and Random Coefficient Approaches, Sankhya: Series B, 59, 346-368.

3. Black, Fischer, 1990, Equilibrium Exchange Rate Hedging, Journal of Finance, 43, 899-908.

4. Bollerslev, Tim, 1986, Generalized autoregressive conditional hetroskedasticity, Journal of Econometrics, 31, 307-327.

5. Bollerslev, Tim, 1990, Modeling the Coherence in Short-Run Nominal Exchange Rates: a Multivariate Generalized ARCH Model, Review of Economics and Statistics, 72, 498-505.

6. Engle, Robert, 1982, Autoregressive conditional heteroskedasticity with estimates of the variance of the U.K inflation, Econometrica, 50, 987-1008.

7. Eun, C., and B. Resnick, 1988, Exchange rate uncertainty, forward contracts and international portfolio selection, Journal of Finance, 43, 197-216.

8. Glen, Jack, and Phillipe Jorion, Currency hedging for international portfolios, Journal of Finance, 48, 1993, 1865- 1886.

9. Kroner, Kenneth, and Jahangir Sultan, 1991, Foreign currency futures and time-varying hedge ratios, in S. Ghon Rhee and Rosita Change, eds., Pacific-Basin Capital Markets Research, vol II, North-Holland, Amsterdam, 397-412. 
10. Kroner, Kenneth, and Claessens, S. 1991, Optimal dynamic hedging portfolios and the currency composition of external debt, Journal of International Money and Finance, 10, 131-148.

11. Levich, Richard \& Lee R, Thomas, 1993, Internationally diversified bond portfolios: the merits of active currency risk management; NBER working paper series.

12. Lioui, Abraham, and Patrice Poncet, 2002, Optimal currency risk hedging, Journal of International Money and Finance, 21, 241-264.

13. Sheedy, Elizabeth, 1998, Correlation in currency markets: a risk-adjusted perspective, Journal of Financial Markets, Institutions and Money 8, 59-82.

14. Solnik, P. 1974, A model of international asset pricing, Journal of Financial Economics, 9 383-406.

15. Tong, Wilson, 1996, An examination of dynamic hedging, Journal of International Money and Finance, 15, 19-35.

16. Tse, Y.K. and Tsui, Albert, 2002, A multivariate generalized Autoregressive Conditional Heteroskedasticity model with time-varying correlations, Journal of Business and Economics Statistics, 20, 351-362.

Notes

Notes 